\title{
The performance of LED calibration system for Cherenkov telescope of LHAASO
}

\author{
M.J.Yang ${ }^{a *}$, S.H.Chen ${ }^{a}$, S.S.Zhang ${ }^{a}$, Y.Zhang ${ }^{a}$, L.Q.Yin ${ }^{a}$, Z.Y.You ${ }^{a, b}$, L.L.Ma ${ }^{a}$, \\ W.M.Zhang ${ }^{a, c}$, Z.Cao $^{a, b}$ for the LHAASO Collaboration \\ ${ }^{a}$ Key Laboratory of Particle Astrophysics, Institute of High Energy Physics, CAS, Beijing, China. \\ ${ }^{b}$ University of Chinese Academy of Science, Beijing, China. \\ ${ }^{c}$ School of Nuclear Science and Technology, Lanzhou University, Lanzhou, China. \\ E-mail: yangmingjiedihep.ac.cn
}

Each SiPM based Cherenkov telescope of LHAASO has an array of $32 \times 32$ SiPMs. SiPM-based camera can be run in the moon night and achieve bout $30 \%$ of duty cycle. The gain of the SiPM varies with the intensity of the sky background light. A UV-LED system is mounted at the center of the mirror to monitor and calibrate the gain of the SiPM-based camera. The LED is also used to monitor the temperature and high voltage compensation loop which is used to keep the SiPM gain stable in the case of environmental temperature changes. LED is working in the constant temperature system. The change of LED luminous intensity with ambient temperature is less than $0.8 \%$. The first two SiPM based Cherenkov telescopes were successfully run in the first half of 2019. The design and performance of LED calibration system, which is a very important tool to monitor the gain stability of the SiPM-based camera, are presented in the paper.

36th International Cosmic Ray Conference -ICRC2019-

July 24th - August 1st, 2019

Madison, WI, U.S.A.

\footnotetext{
* Speaker.
} 


\section{Introduction}

This paper introduces a UV-LED calibration system developed for WFCTA(Wide Field of view Cherenkov Telescope Array) of LHAASO (Large High Altitude Air Shower Observatory), which is built at Mt. Haizi (4410 m asl, N29 $21^{\prime} 31^{\prime \prime}$, E100 $\left.{ }^{\prime} 1^{\prime} 5^{\prime \prime}\right)$ in Sichuan Province, China [1]. Based on the joint observation of three detector arrays:a square kilometer array (KM2A), water Cherenkov detector array (WCDA) and wide field of view Cherenkov telescope array (WFCTA), LHAASO is aimed at exploring the origin of high-energy cosmic ray [2].WFCTA measures the cosmic ray energy through detecting the Cherenkov light caused by secondary charged particle in air showers initiated by cosmic rays with the energy range from $10^{13} \mathrm{eV}$ to $10^{18} \mathrm{eV}$ [3].

WFCTA consists of SiPM-based camera, electronics, reflective spherical optical system, control system, calibration and monitor system. Compared with PMT (Photomultipliers Tube), SiPM (Silicon Photomultipliers) has some advantages, like lower power voltage, higher photon detection efficiency, more compact structure, and no death or aging to strong light exposure, which make SiPM a better choice for WFCTA, especially when it could work normally under moon light, which could increase the duty cycle from $10 \%$ to $30 \%$ [4].

Due to the thermal electron motion, SiPM's breakdown voltage increases with the rise of temperature. While, SiPM's gain is proportional to the overvoltage which is the difference between SiPM's bias voltage and breakdown voltage (as shown in function( 1.1)). As a result, SiPM's gain would decrease with the rise of temperature [5], [6]. On the other hand, the protective resistance, mounted between SiPM's cathode and the power source to protect SiPM from strong light exposure, could influence SiPM's gain when the background light changes.

$$
G_{\text {SiPM }}=\frac{Q}{e}=\frac{C}{e}\left(V_{\text {bias }}-V_{\text {breakdown }}\right)=\frac{C}{e}\left(V_{\text {bias }}-V_{\text {breakdown }}^{T_{0}}-\kappa T\right)
$$

where $\mathrm{Q}$ is the SiPM output charge when fired by a photon; e is the electron charge, $1.602 \times 10^{-19} \mathrm{C}$; $\mathrm{V}_{\text {bias }}$ is the bias voltage added to the cathode of SiPM; $\mathrm{V}_{\text {breakdown }}$ is the breakdown voltage for SiPM; $\mathrm{T}$ is the ambient temperature for SiPM; and $\kappa$ is the temperature factor of SiPM.

In order to monitor and calibrate SiPM's gain under varying ambient temperature and background light, a UV-LED flasher system is developed. Considering the fact WFCTA would work at two modes: Cherenkov-light mode and fluorescent-light mode, the duration time of light pulse could be changed from 10ns to $10 \mu \mathrm{s}$. The frequency of UV-LED light pulse is also adjustable to obtain high statistics in short time and only limited by electronics or the observation plan of WFCTA. Moreover, due to the temperature dependency of LED's light output, a temperature feedback circuit is employed to keep LED working in constant temperature environment with the temperature variation of $\pm 0.2{ }^{\circ} \mathrm{C}$.

\section{UV-LED system design}

The UV-LED assembly is shown in Figure 1. LEDs and temperature feedback circuit board are fixed at the back of black tube with the length of $15 \mathrm{~cm}$ and diameter of $10 \mathrm{~cm}$ for light output, while a piece of Teflon is mounted at the front of the tube to spread the LED light to be a uniform surface light source. LEDs are driven and controlled by pulses generated by a CMOS gate driver (IXRFD630) transmitted through coaxial cables. 


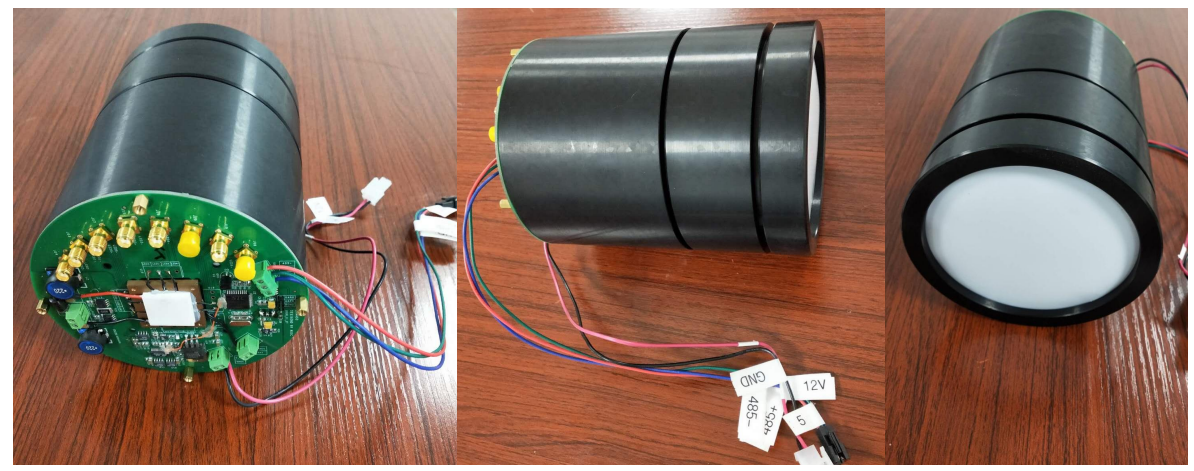

Figure 1: UV-LED assembly (Left: rear view; Center: side view; Right: front view).

As shown in Figure 2, the CMOS gate driver is powered by a DC linear power supply (VCC) varying from $3.3 \mathrm{~V}$ to $30 \mathrm{~V}$ and controlled by a TTL gate signal (TTL) generated by FPGA in the logic circuit board. The shape of LED light is determined by the amplitude and width of pulses generated by the COMS gate driver, further depending on the level of VCC and width of TTL fundamentally. The flashing rate could be regulated trough changing the frequency of TTL signal, and the intensity of a signal pulse could be controlled by the VCC level, the TTL width and the number of LEDs flashed.

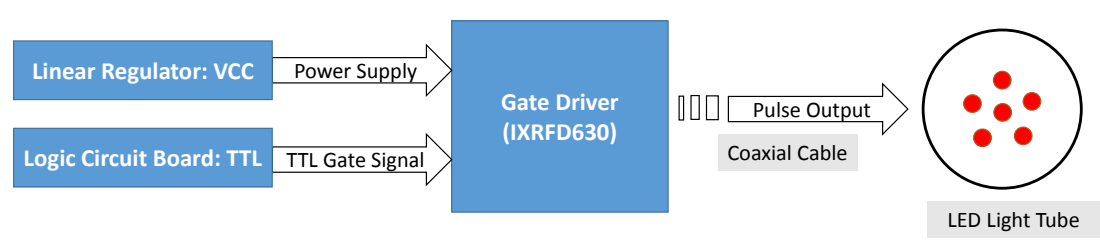

Figure 2: Functional diagram for UV-LED

In practice, we adjust the LED light intensity only through changing the TTL width and the number of LEDs flashed for reduction procedure. TTL signal width could be adjusted from $10 \mathrm{~ns}$ to $10 \mu \mathrm{s}$ to make the shape of LED light pulse be similar with both the Cherenkov light and the fluorescent light. Considering the temperature dependency of LED's light output, a temperature feedback circuit is employed and a copper billet is mounted on the bottom of LEDs.Depending on the temperature feedback from the temperature sensor, situated between the copper billet and LEDs, the temperature feedback circuit modulates LEDs' temperature through heating the copper billet. To obtain better temperature control effect, the rear cover, foam and Teflon are employed to build a narrow temperature space for LEDs and the copper billet.

To monitor the gain character of SiPM-based camera for WFCTA, UV-LEDs is mounted at the center of the spherical reflective mirror. Moreover, the communication is built based on RS485 interface. With the help of a serial server, computer could visit the UV-LED system through the local network covering the observation station at Mt. Haizi based on TCP/IP standard. A simple data acquisition program is developed based on LabWindows/CVI to control and monitor the working 
status of the UV-LED system.
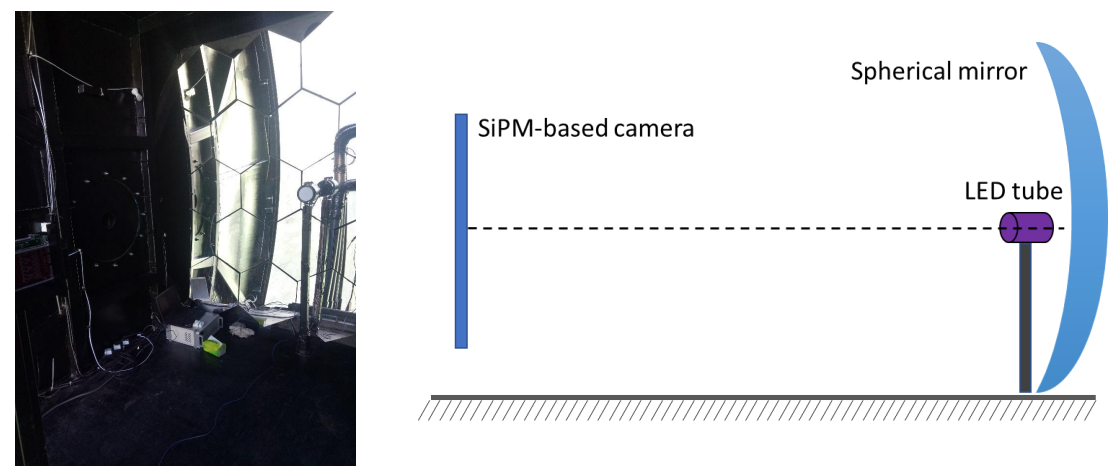

Figure 3: UV-LED mounted position

\section{UV-LED system test}

The UV-LED system was tested using a temperature regulating chamber, a SiPM-based subcluster, which was a part of SiPM-based camera, and trigger electronics. Firstly, we evaluated the stability of the test platform; secondly, the UV-LED system's stability about temperature and light intensity were tested; and then, the distribution of LED light intensity at the camera's incident plane for WFCTA's worksite was scanned and analyzed.

\subsection{Stability of test platform}

Apart from the temperature dependency for SiPM, there were still some potential unstable factors influencing the stability of test platform, like power supply, communication, and electronic characteristics and so on. So evaluating the stability of test platform was necessary before evaluating the stability of the UV-LED system.

For evaluation, both the SiPM-based subcluster and UV-LED system were put inside the temperature chamber which provided a constant temperature environment of $20^{\circ} \mathrm{C}$. SiPMs were illuminated by the light pulse with 20ns-width, $200 \mathrm{~Hz}$-frequency and $405 \mathrm{~ns}$-peak wavelength emitted from the UV-LED. The LED response signal and temperature measuring data generated by the subcluster were transmitted through Internet cables based on TCP/IP standard.

During the evaluation, the UV-LED system was ordered to control the LED's temperature to $40^{\circ} \mathrm{C}$, while LED's actual temperature was controlled to $42.2 \pm 0.2^{\circ} \mathrm{C}$ (as shown in Figure 4) measured by a temperature sensor mounted at the bottom of LED. Some points much higher or lower than $42.2^{\circ} \mathrm{C}$ were resulted from the electromagnetic interference added to the temperature measurement signal. The difference between the set temperature and the constant temperature was due to the no-calibration for the temperature sensor and the no-calibration for modulation point of the temperature feedback circuit. While, compared with the constant temperature value, the constant temperature effect was much more important to the UV-LED's stability.

Even though the subcluster worked in the constant temperature environment, SiPM' s temperature still varied slowly from $22.3^{\circ} \mathrm{C}$ to $22.9^{\circ} \mathrm{C}$ during the 720 minutes because of electronic 

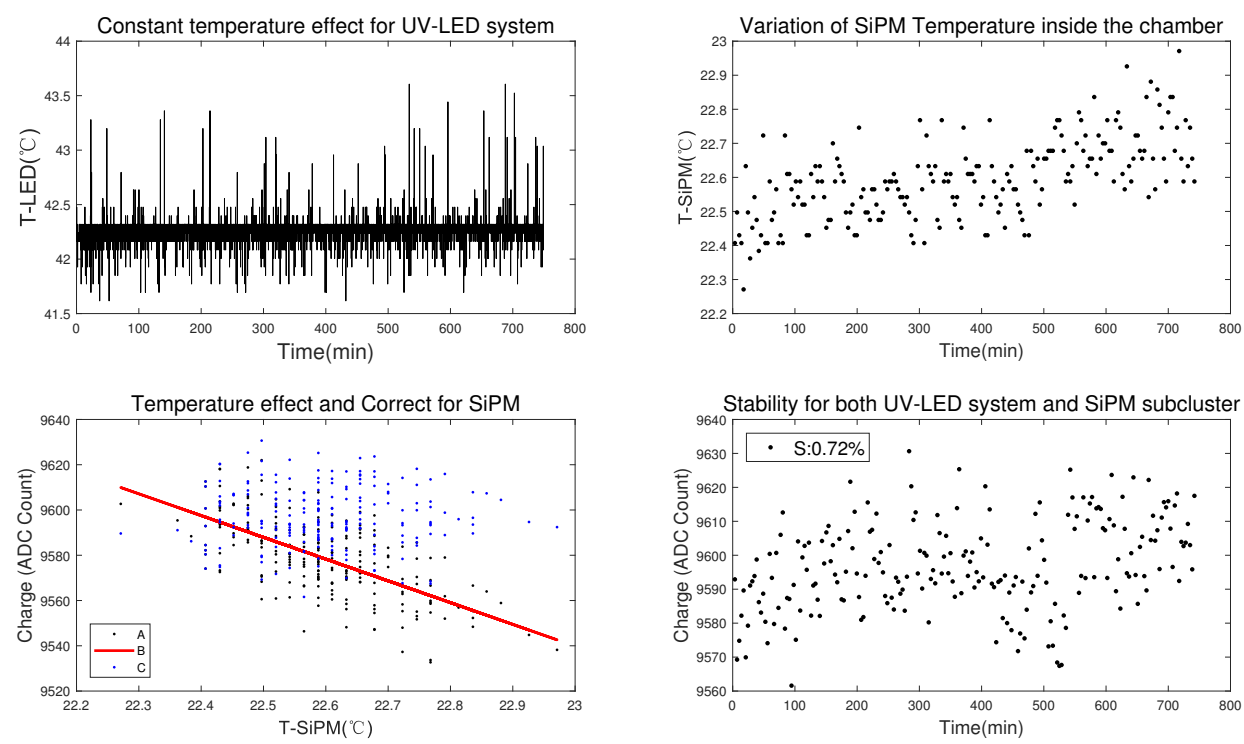

Figure 4: Stability evaluation for both the UV-LED system and the SiPM-based subcluster at $20^{\circ} \mathrm{C}$ for 720 minutes (A: original signal data with temperature dependency; B: fitting curve for temperature dependency; C: signal data with temperature correction)

heating (as shown in Figure 4). Considering the temperature dependency of SiPM's gain, a linear function, based on the fitting result about the relationship of the measurement signal charge and SiPM's temperature, was added to the origin data to correct the temperature effect. Moreover, the phenomenon of temperature effect trend almost had nothing to do with the change of UV-LED's temperature, since LED's light intensity would increase with the rise of temperature, and LED's temperature was kept to a constant about $42.2 \pm 0.2^{\circ} \mathrm{C}$.

After the correction for SiPM's temperature dependency, the measured signal charge for LED light pulse emitted by the UV-LED system varied randomly within 720 minutes with the variation range under $0.8 \%$, when both the UV-LED system and SiPM-based subcluster worked inside a same temperature chamber at $20^{\circ} \mathrm{C}$ (as shown in Figure 4). This evaluation could present the stability for both the UV-LED system and the SiPM-based subcluster in normal operational environment but the variation of temperature. The variation range called $S$ was calculated by function( 3.1).

$$
S=\frac{\left(\text { Signal }_{\text {max }}-\text { Signal }_{\text {min }}\right)}{\text { Signal }_{\text {mean }}} \times 100 \%
$$

\subsection{Stability of UV-LED system}

To evaluate the stability of UV-LED under variable ambient temperature similar to the actual working environment at Mt. Haizi, we put UV-LED system inside the temperature chamber for changing its environmental temperature and placed the subcluster inside a dark box in the lab where the temperature is controlled to $26^{\circ} \mathrm{C}$ by the air conditioner. LED light pulse was sent to SiPMs through an optical fiber. 
During the test, the temperature chamber worked at a program that changed its temperature from $-20^{\circ} \mathrm{C}$ to $30^{\circ} \mathrm{C}$ in 90 minutes, while the UV-LED system was ordered to control the LED's temperature to $40^{\circ} \mathrm{C}$. As shown in Figure 5, LED's temperature was controlled to $42.2 \pm 0.2^{\circ} \mathrm{C}$ measured by a temperature sensor mounted at the bottom of LED, and there was no obvious difference from the temperature curve of Figure 4 that was obtained at the constant temperature condition of $20^{\circ} \mathrm{C}$.
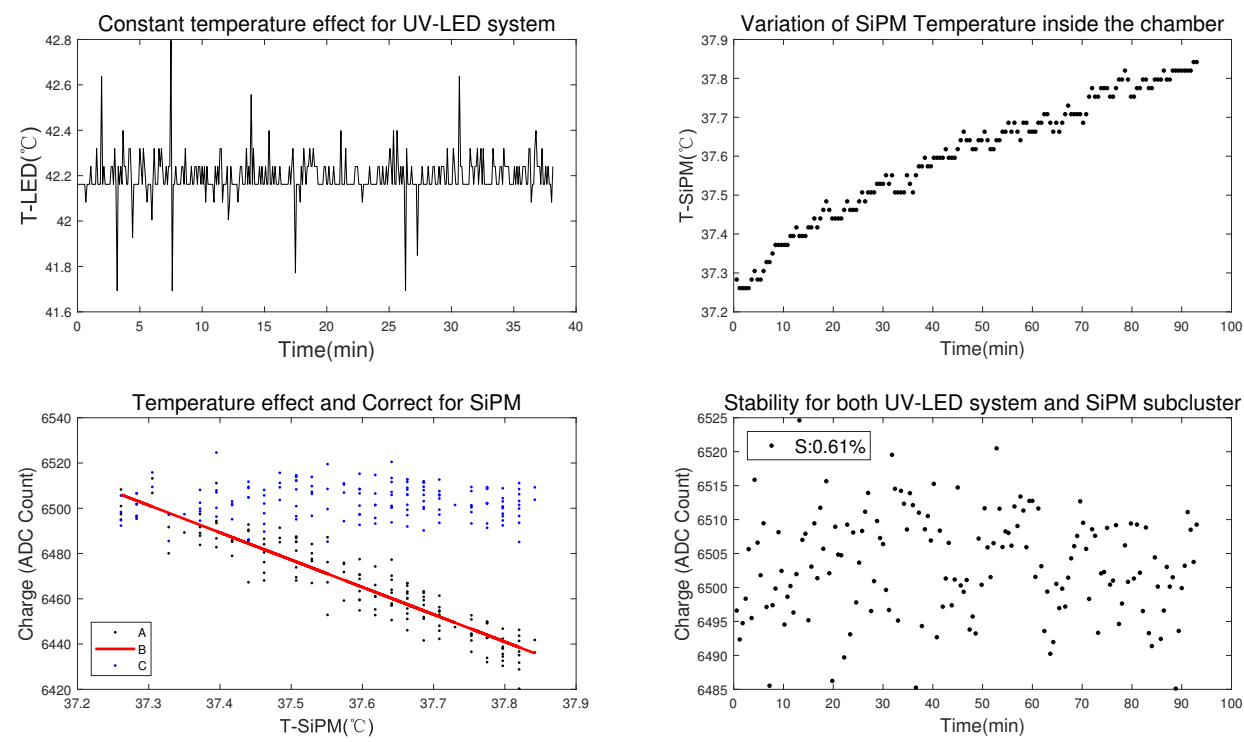

Figure 5: Stability evaluation for UV-LED system under variable temperature environment from $-20^{\circ} \mathrm{C}$ to $30^{\circ} \mathrm{C}$ when controlling temperature to $40^{\circ} \mathrm{C}$ (A: original signal data with temperature dependency; B: fitting curve for temperature dependency; $\mathrm{C}$ : signal data with temperature correction)

The LED light pulse emitted from the UV-LED system was set to $200 \mathrm{~Hz}$-frequency and 60nswidth. Working inside a dark box in the lab, SiPMs' temperature, which increased from $37.2^{\circ} \mathrm{C}$ to $37.9^{\circ} \mathrm{C}$ in 90 minutes, changed much faster than in the constant temperature provided by the temperature chamber(as shown the ch1 in Figure 5 and Figure 4).

The correction for SiPM's temperature dependency was executed as described in section 3.1. After the correction, the measurement signal charge for LED light pulse emitted by the UV-LED system varied randomly within 90 minutes with the variation range under $0.8 \%$ and there was no obvious difference from the stability presented in section 3.1, when the UV-LED's environmental temperature varied from $-20^{\circ} \mathrm{C}$ to $30^{\circ} \mathrm{C}$.

\subsection{Distribution of LED light intensity}

When the UV-LED system was mounted at Mt. Haizi (as shown in Figure 3 ), we fixed the SiPM-based subcluster at a bracket, which could move at $\mathrm{X}$ and $\mathrm{Y}$ direction with the range of $1000 \mathrm{~mm} \times 1000 \mathrm{~mm}$ and the precision of $1 \mathrm{~mm}$, to scan and test the distribution of LED light intensity near the plane of SiPM-based camera.

For normalizing the gain for $16 \mathrm{SiPMs}$ arranged as $4 \times 4$ in the subcluster, we moved the bracket with the step of $25.8 \mathrm{~mm} 4$ times respectively at $\mathrm{X}$ and $\mathrm{Y}$ direction, which was equal to 
the center interval between each two SiPMs. As a result, we could normalize each SiPM's gain to the rightest SiPMs, and then we could normalize all the SiPM's gain to the SiPM at bottom right corner. Moreover, even we choose SiPMs at the same gain for the subcluster, there was still some difference among each channel because of the difference among each electronic channel. So the normalization for each SiPM or channel in the subcluster was necessary.
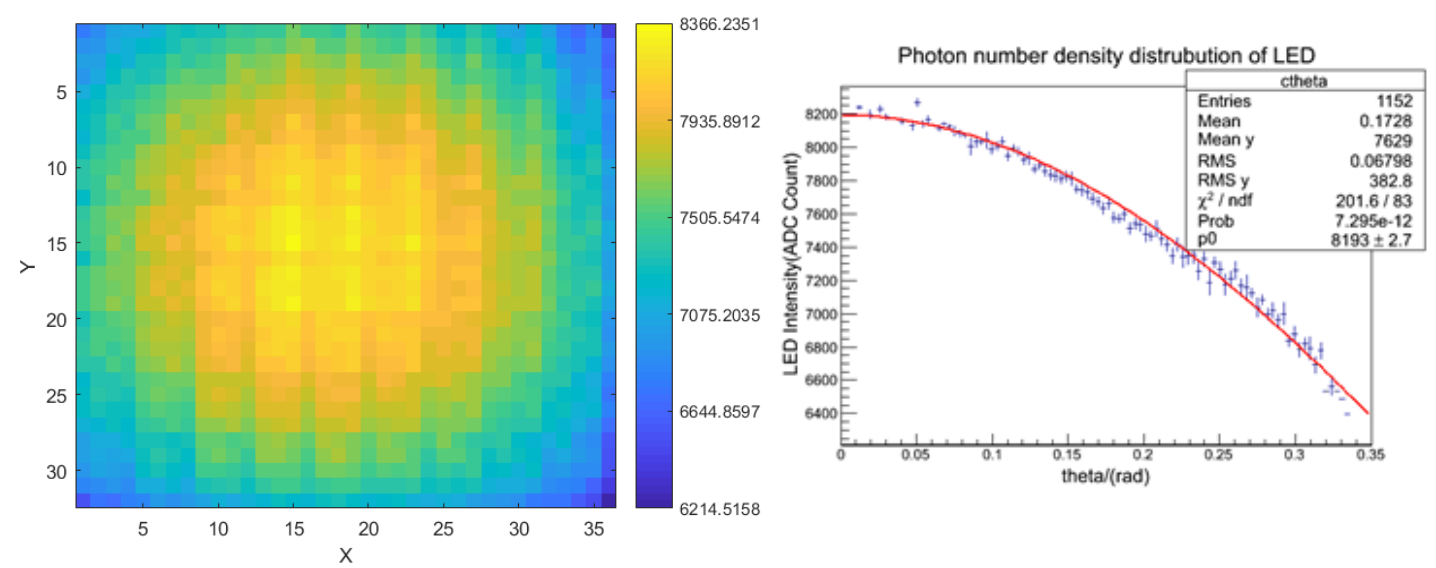

Figure 6: 2D-distribution of LED light intensity at scanned plane (left) and the distribution rule for the LED light intensity(right)

Combining the maximum detection area for the subcluster with the test efficiency, the subcluster was moved with the step of $110 \mathrm{~mm} 7$ times at $\mathrm{Y}$ direction and $100 \mathrm{~mm} 8$ times at $\mathrm{X}$ direction and the scanned area was about $900 \mathrm{~mm} \times 900 \mathrm{~mm}$.

A 3D relative coordinate was built to include both the UV-LED and the scanned area. Thanks to the Teflon in front of LEDs, the UV-LED system could be regard as a surface light source. The distribution of LED light intensity at the canned plane was shown in Figure 6 and the light intensity at each point obeyed the rule of $\cos \theta^{4}$, where $\theta$ was the angle between the connection from the center of LED to the center of SiPM and the perpendicular connection form LED light emitting pane to the canned plane [7].

\section{Conclusion}

Developed for monitoring and calibrating the gain of SiPM-based camera for WFCTA, the UVLED system was capable to make its light pulse width vary from $10 \mathrm{~ns}$ to $10 \mu \mathrm{s}$, while its flashed frequency was only limited by the maximum transfer rate for data acquisition system and electronics. Moreover, thanks to the temperature feedback circuit, the intensity fluctuation of LED's light pulse could be controlled below $0.8 \%$ under the variable ambient temperature from $-20^{\circ} \mathrm{C}$ to $30^{\circ} \mathrm{C}$.

Considering the temperature dependency for LED light intensity and the fact that there was no temperature calibration for the temperature feedback circuit used to control LED's temperature, each UV-LED's light intensity should be calibrated at the same temperature value which would be 
set for the temperature control for LEDs based on the same test platform like the same temperature chamber and the same SiPM-based subcluster.

\section{Acknowledgements}

This work is supported in China by the Key Laboratory of Particle Astrophysics, Institute of High Energy Physics, CAS. Projects No. 2018YFA0404201 and No. 2018YFA0404202 of the National Key R\&D Program of China and Projects No. 11475190 and No. 11675204 of NSFC also provide support to this study.

\section{References}

[1] Z. Cao, Chinese Phys. C 34 (2010) 249-252.

[2] H. He, LHAASO Project: detector design and prototype, in: Proceedings of the 31st ICRC, Lodz, Poland, 2009.

[3] Hanna D, Nuclear Instruments\& Methods in Physics Research, 2010, 612(2):278-287.

[4] Baiyang B, Nuclear Instruments and Methods in Physics Research Section A: Accelerators, Spectrometers, Detectors and Associated Equipment, 2018, 899:94-100.

[5] Ramilli M, Nuclear Science Symposium Conference Record, IEEE, 2008.

[6] Yang M J, Review of Scientific Instruments, 2016, 87(11):113308.

[7] Zhang S S, Nuclear Inst \& Methods in Physics Research A, 2011, 629(1):57-65. 